\title{
A Survey of the Key Technologies of Heterogeneous Wireless Converged Network
}

\author{
Xiaoxue Guo ${ }^{1, a}$, Rong $\mathrm{Li}^{2, \mathrm{~b}}$, Huomin Liang ${ }^{3, \mathrm{c}}$ \\ ${ }^{1}$ Guangdong University of Petrochemical Technology, College of Science, Maoming, China \\ ${ }^{2}$ Guangdong University of Petrochemical Technology, College of Science, Maoming, China \\ ${ }^{3}$ Guangdong Pharmaceutical University, College of Medical Information Engineering, Guangzhou, \\ China

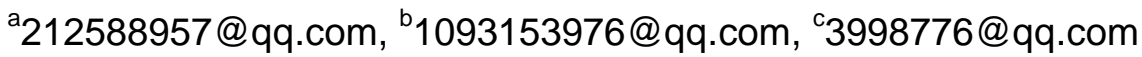

Keywords: heterogeneous; converged networks; resource management; vertical handover;

\begin{abstract}
Heterogeneous Wireless Converged Networks (HWCN) are expected to be the trends of next generation wireless network. It will pose great impacts on way of communication. HWCN is a brand new research area and many problems still need to be solved. Firstly, the background and development of HWCN are summarized, and its challenges and analyzed. Then from the perspective of network architecture, access selection algorithms, resource management algorithms and vertical handover strategies are discussed. Finally, the next step research suggestions of load balancing, green communications and intelligent characteristics are also proposed.
\end{abstract}

\section{Introduction}

Heterogeneous network convergence is the development direction of next generation wireless communication network, and is the result of the evolvement of wireless communication technology, the increase of communication service type and quantity for subscribers and the promotion of market demand[1]. It provides secure, reliable and real-time communication services by overlaying wireless access techniques, and the synergy and convergence between heterogeneous networks is to integrate and use these networks efficiently, not overlaying wireless access techniques simply[2]. Achieving a high network convergence needs to involve technical convergence at different levels of networks, and the heterogeneous networks that achieve such convergence will meet technical challenges, so relevant research institutions and organizations for standardization at home and abroad conduct intensive studies and standardization work on the key techniques in the field of heterogeneous wireless converged networks. And heterogeneous network convergence has gradually become one of the hot research fields throughout the world.

\section{Challenges of heterogeneous wireless converged networks}

\section{Converged Network Architecture.}

In heterogeneous converged networks, the heterogeneity of networks and the diversity of their service types put forward higher requirements on converged network architecture, so it is necessary to design a secure, manageable and controllable heterogeneous converged network architecture which can provide personalized and diversified services for users, and can integrate the foundation structure of backward compatible wireless terminal equipment. How to fully mine and use the heterogeneous characteristics of networks, how to design the converged architecture of backward compatible heterogeneous network and how to resolve the effective interworking and efficient convergence of heterogeneous networks have become key problems in the research field of heterogeneous network convergence[3].

\section{Access selection.}

In the future communication system, there are an increasing number of application contexts in which subscribers can share multiple available networks simultaneously. Due to diversified 
subscriber services, different personal information and diverse network performance, how to select a suitable network as a bearer of current application for subscribers becomes a key problem to guarantee the QoS of subscribers[4]. Currently, most of plans adopt multi-target optimization method. This method, although, has been greatly improved compared with traditional single-target selection, utility function evaluation and other methods, it still lacks universal property, is difficult to comprehensively and rationally evaluate network performance, and can not meet the demands of subscribers and networks.

\section{Vertical handover.}

Horizontal handover between homogeneous networks can be defined by each organization for standardization, while vertical handover technique is the foundation to achieve heterogeneous wireless converged network operation, that is, it ensures that subscribers can make a switch in heterogeneous access techniques on the premise of keeping current session according to wireless environment and type of service[5]. Vertical handover, due to involving different operators, network architectures and bearer modes, still has high cutting off rate and loss rate as well as long delay in applying traditional horizontal handover technique to heterogeneous wireless networks. Therefore, it is necessary to study more flexible and efficient vertical handover mechanisms and performance optimization algorithms to meet mobile subscribers' demands on high transmission speed and high-quality service experience.

\section{Radio resource management.}

RRM (Radio Resource Management) in heterogeneous networks needs to coordinate resources of different access networks, improve compatibility of protocol, and provide QoS guarantee crossing heterogeneous networks[6]. Meanwhile, with abundant types of service and increasing application demands, especially with the emergence of high-bandwidth service demands[7], people require more and more wireless network resources, and the contradiction between increasing resource demand and limited network resource supply has become one of the main factors to restrict the development of wireless communication. Therefore, it becomes one of the technical challenges that need to be resolved to rationally and efficiently make use of wireless network resources for heterogeneous network convergence.

\section{End-to-end QoS guarantee.}

Providing QoS guarantee is to meet different service level agreements achieved with various subscribers by adopting suitable measures, and to optimize QoS parameters[8], such as serviceability, time delay, jitter, throughput capacity, and loss rate. Currently, the research on the QoS of most wireless networks mainly focuses on homogeneous wireless access networks. End-to-end QoS guarantee in heterogeneous networks is important both for the resource utilization of optimal heterogeneous networks and for the design of collaborative work between access networks[9]. In such background, how to guarantee end-to-end QoS for subscriber services in heterogeneous converged networks remains to be studied intensively.

\section{Correlation research}

\section{Access Selection and Admission control Algorithms.}

Traditional Access Selection and Admission Control algorithms mainly include RA (Random Access), SAW (Simple Additive Weighting), MEW (Multiplicative Exponent Weighting), HBP (High Bandwidth Precedence), TOPSIS (Technique for Order Performance by Similarity to Ideal Solution), and GRA (Grey Relational Analysis)[10], most of which are based on static network parameters, without considering the dynamic and variation trend of these parameters.

Research on modified Access Selection and Admission Control Algorithms mainly includes the following types: The first type is the algorithm based on type of service which selects network or controls admission according to the type of each request call. The fundamental point of this algorithm is that different wireless access techniques have their individual characteristics and that types of services suitable to be supported also differ, so it selects the most efficient network that can guarantee 
QoS demands of subscribers to access. But this algorithm also easily causes the imbalance of load between networks; The other type is the algorithm based on utility function/cost function which selects target network to optimize resource allocation through maximal access utility or minimum access cost $[11,12]$.

Generally speaking, existing access selection and admission control algorithms always have incogitant access judgment index, strong fixed-subjectivity of parameters or weight, complex calculation and low universal property. Although these algorithms, to a certain extent, can improve system performance, they still have larger space to be optimized.

\section{Resource Management Architecture and Algorithms.}

Compared with traditional wireless network resource management, due to influences of multiple network resource forms in heterogeneous wireless converged network system, various service requirements for subscribers and different commercial operation modes, RRM in heterogeneous networks consists of the collection of a set of different wireless network control mechanisms[13]. According to different resource management strategies, the research on heterogeneous wireless network resources is divided into the following three types:

The first type is the resource allocation algorithm based on user utility optimization which always selects the service parameters related to QoS of subscriber services to evaluate network performance, such as received signal intensity, bit error rate, access bandwidth, price and etc, and allocates resources on the premise of optimal system user utility[14, 15]; The second type is the algorithm based on system utility maximization which maximizes system interests through the allocation and distributed plans of services and the cooperative transmission service flow between multiple networks, such as power saving maximization, network system throughput maximization and network capacity utilization rate maximization; The third type is the algorithm based on fairness which quantizes the fairness of resource allocation through standardized formula and guarantees the fairness for different users, services and networks through mechanism, but other algorithms always cause damage to part of users' fairness in improving system throughput and reducing time delay.

\section{Vertical Handover Strategies.}

Currently, most of the research on vertical handover mainly focuses on handover decision and handover execution.

With regard to existing research, vertical handover technique still has the following shortages in performance[16], handover decision and inter-operational control. The first aspect is optimization of performance. Vertical handover must be achieved at high layer in order to ensure the independence with bottom layer access technique, but universal property at high layer may cause damage to network performance inevitably[17]. The second aspect is efficient and feasible handover decision method. Vertical handover decision is a typical multi-standard decision problem, but existing research has poor feasibility and ping-pong effect, and is not conductive to effective using of network resources. The third aspect is interoperability in vertical handover which is used to handle the differences of access techniques in QoS, AAA and other control techniques.

\section{Future research directions}

\section{Load Balancing.}

Load balancing, an effective way to improve quality of network operation, can be used to relieve or resolve imbalance of resource distribution in heterogeneous converged networks[18, 19]. The research on load balancing in heterogeneous wireless converged networks is to select the most suitable network to serve new services based on some strategies of access selection and admission control, which avoids differences in load caused by unbalanced service arrival, improving system capacity, QoS and increasing radio resource utilization rate.

\section{Green Communications.}

In the development of wireless access networks, multi-standard heterogeneous networks overlay intensively, and a lot of base station is in the working condition of non-full load, which causes a low 
radio resource utilization rate and a huge waste of energy consumption inevitably[20]. In view of less research on cooperative energy saving of base station in heterogeneous networks, the design objective of green communications network system is to reduce energy consumption of the system on the premise of meeting the QoS for users within networks[21]. Therefore, how to achieve efficient green communications to save the energy of wireless communication networks is one of the problems that need to be resolved in the future.

\section{Intelligent Characteristics.}

Current heterogeneous wireless converged networks lack intelligence or only have part of intelligence, and network function entities lack self-perception, self-configuration, self-learning and other performances, so that these networks can not adapt to more complex, heterogeneous and dynamic network environment[22]. In such environment, simple static configuration can not meet diversified needs, and simple integration and compromise always make various users unable to obtain satisfying services. Therefore, it is necessary to introduce intelligent characteristics into heterogeneous converged networks to study new network architecture and behavior model, and it is also necessary to greatly improve key techniques, which need to be further studied carefully[23].

\section{Conclusions}

Next-generation wireless communication system will be a ubiquitous IP converged network which supports multiple access techniques, provides diversified services and has intelligent characteristics. The objective of converged techniques is to provide unified converged services in heterogeneous wireless networks, and the proposal of network convergence is to mask the heterogeneity of bottom layer network technique at different layers technically. Therefore, heterogeneous converged networks have a good prospect for research and development in academic sector and multi-industry application area.

This paper presents the relevant background and development of heterogeneous wireless converged networks, discusses the challenges to these networks, summarizes the researches on such key technologies in these networks as interworking architecture, access selection, admission control, radio resource management as well as vertical handover, analyzes problems in these researches, and puts forward suggestions on further research and improvement in view of these problems.

\section{References}

[1]Gulyani M. Converged All-IP Networks Maximize Services[J]. ENRICHING COMMUNICATIONS. 2011, 5(1).

[2] Hu Y H, Shou G C, Qian Z J, et al. Research on Converged Access Network of WDM-PON and WiMAX[J]. INFORMATION TECHNOLOGY FOR MANUFACTURING SYSTEMS II, PTS 1-3. 2011, 58-60: 1583-1589.

[3] Gercheva R S, Chitti S H, Prasad N R, et al. Review On the Benefits of Converged Next Generation Network Architecture[J]. 2013 16TH INTERNATIONAL SYMPOSIUM ON WIRELESS PERSONAL MULTIMEDIA COMMUNICATIONS (WPMC). 2013.

[4] Haldar K L, Ghosh C, Agrawal D P. Dynamic spectrum access and network selection in heterogeneous cognitive wireless networks[J]. PERVASIVE AND MOBILE COMPUTING. 2013, 9(4): 484-497.

[5] Abdullah R M, Abdullah A, Hamid N, et al. The Rapid Vertical Handover for Efficient IPv6 Mobility Support in Heterogeneous Wireless Networks[J]. ARABIAN JOURNAL FOR SCIENCE AND ENGINEERING. 2014, 39(2): 851-860. 
[6] Castillo-Lema J, Cruz E, Neto A, et al. Advanced Resource Provisioning in Context-Sensitive Converged Networks[J]. 2013 INTERNATIONAL CONFERENCE ON COMPUTING, NETWORKING AND COMMUNICATIONS (ICNC). 2013.

[7] Harounabadi M, Sedighi M. A Fast Response Dynamic Bandwidth Allocation Algorithm for a Converged EPON and WiMAX Network[J]. 2012 SIXTH INTERNATIONAL SYMPOSIUM ON TELECOMMUNICATIONS (IST). 2012: 470-476.

[8] Lim W S, Kanonakis K, Kourtessis P, et al. Flexible QoS Differentiation in Converged OFDMA-PON and LTE Networks[J]. 2013 OPTICAL FIBER COMMUNICATION CONFERENCE AND EXPOSITION AND THE NATIONAL FIBER OPTIC ENGINEERS CONFERENCE (OFC/NFOEC). 2013.

[9] Ho D, Park Y, Song H. QoS-supporting video streaming system with minimum data service cost over heterogeneous wireless networks[J]. JOURNAL OF VISUAL COMMUNICATION AND IMAGE REPRESENTATION. 2013, 24(8): 1293-1302.

[10] Shen W, Zeng Q A. Cost-Function-Based Network Selection Strategy in Integrated Wireless and Mobile Networks[J]. IEEE TRANSACTIONS ON VEHICULAR TECHNOLOGY. 2008, 57(6): 3778-3788.

[11] El Helou M, Lahoud S, Ibrahim M, et al. Satisfaction-based Radio Access Technology Selection in Heterogeneous Wireless Networks[J]. 2013 IFIP WIRELESS DAYS (WD). 2013.

[12]Smaoui I, Zarai F, Bouallegue R, et al. Multi-Criteria Dynamic Access Selection in Heterogeneous Wireless Networks[J]. 2009 6TH INTERNATIONAL SYMPOSIUM ON WIRELESS COMMUNICATION SYSTEMS (ISWCS 2009). 2009: 338-342.

[13] Li W, Delicato F C, Pires P F, et al. Efficient allocation of resources in multiple heterogeneous Wireless Sensor Networks[J]. JOURNAL OF PARALLEL AND DISTRIBUTED COMPUTING. 2014, 74(1): 1775-1788.

[14] Shi Z, Zhu Q. Radio Resource Management Scheme for Heterogeneous Wireless Networks Based on Access Proportion Optimization[J]. JOURNAL OF COMMUNICATIONS AND NETWORKS. 2013, 15(5): 527-537.

[15] Ismail M, Zhuang W H. A Distributed Multi-Service Resource Allocation Algorithm in Heterogeneous Wireless Access Medium[J]. IEEE JOURNAL ON SELECTED AREAS IN COMMUNICATIONS. 2012, 30(2): 425-432.

[16] Li L M, Ma L, Xu Y B, et al. Motion Adaptive Vertical Handoff in Cellular/WLAN Heterogeneous Wireless Network[J]. SCIENTIFIC WORLD JOURNAL. 2014(341038).

[17] Song Y, Kong P Y, Han Y. Power-Optimized Vertical Handover Scheme for Heterogeneous Wireless Networks[J]. IEEE COMMUNICATIONS LETTERS. 2014, 18(2): 277-280.

[18] Ali M, Pillai P, Hu Y F, et al. Fuzzy Based CRRM for Load Balancing in Heterogeneous Wireless Networks[J]. PERSONAL SATELLITE SERVICES, PSATS 2013. 2013, 123: 135-145.

[19] Pourmina M A, Mirmotahhary N. Load balancing algorithm by vertical handover for integrated heterogeneous wireless networks[J]. EURASIP JOURNAL ON WIRELESS COMMUNICATIONS AND NETWORKING. 2012(14).

[20] Navaratnarajah S, Saeed A, Dianati M, et al. ENERGY EFFICIENCY IN HETEROGENEOUS WIRELESS ACCESS NETWORKS[J]. IEEE WIRELESS COMMUNICATIONS. 2013, 20(5): 37-43. 
[21] Ranaweera C, Lim C, Wong E, et al. Energy Consumption of Next-Generation Optical-Wireless Converged Networks[J]. 2012 ASIA COMMUNICATIONS AND PHOTONICS CONFERENCE (ACP). 2012.

[22] Yuan F C, Chen Y M, Chung T Y. Intelligent call setup strategy for multimedia communication in heterogeneous wireless network[J]. EXPERT SYSTEMS WITH APPLICATIONS. 2012, 39(1): 1298-1305.

[23] Wen J, Sheng M, Zhang Y, et al. Traffic Characteristics Based Dynamic Radio Resource Management in Heterogeneous Wireless Networks[J]. CHINA COMMUNICATIONS. 2014, 11(1): 1-11. 